SECCIÓN I * TEMA CENTRAL

Instituciones y gobernabilidad 



\title{
Acuerdos de gobernabilidad para la sostenibilidad: una perspectiva regional*
}

\author{
Philippe C. Schmitter**
}

\begin{abstract}
Resumen
Propósito

El artículo se centra en las precondiciones para forjar estrategias internacionales de modo de lidiar con la sostenibilidad social y ambiental.

Considerando el Informe Brundtland y su estrategia implícita basada en conferencias intergubernamentales y tratados a nivel global como punto de partida, este artículo sugiere una estrategia alternativa que se enfoca en los acuerdos de gobernabilidad (AGs) en lugar de en los organismos de gobierno y en el nivel regional más bien que en el global.

El artículo argumenta que para que éstos funcionen efectivamente, ellos deben ser percibidos como legítimos por quienes son afectados por sus decisiones.

Este artículo sostiene que esto dependerá de su conformidad con ciertos principios genéricos que regulan la constitución, composición y la toma de decisiones de tales AGs. Si éstos son exitosos en Europa, sus normas pueden ser difundidas a otras regiones del mundo.
\end{abstract}

\section{Diseño/metodología/aproximación}

Este es un artículo conceptual que presenta principios para una gobernabilidad internacional para la sostenibilidad basados en experiencias actuales con la gobernabilidad en la Unión Europea (UE).

\section{Hallazgos}

El artículo apunta que desde la publicación del Informe Brundtland, entre los cambios políticos/ institucionales más significativos con respecto a las políticas para la sostenibilidad ambiental han estado la expansión de las competencias de la UE y el surgimiento de un numeroso y amplio conjunto de organizaciones transnacionales. El artículo muestra que este nuevo terreno institucional puede ser utilizado para forjar nuevas estrategias de gobernabilidad para la sostenibilidad.

\section{Originalidad/valor}

La originalidad/valor del artículo yace en su análisis de la toma de decisiones en la UE como punto de partida de la gobernabilidad global para la sostenibilidad.

Palabras clave: Gobernabilidad - Unión Europea - sostenibilidad.

* Traducción de Claudia Baeza y Javier Loyola.

** Instituto Universitario Europeo. 


\begin{abstract}
Purpose

The article focuses on the preconditions for forging International strategies to deal with social and environmental sustainability.

Taking the Brundtland Report and its implied strategy based on inter-governmental conferences and treaties at the global level as a point of departure, this article suggests an alternative strategy which focuses on governance arrangements (GAs) rather than government agencies and at the regional rather than the global level.

The article argues that for these to function effectively, they must be perceived as legitimate by those affected by their decisions.

This article argues that this will depend on their conformity to certain generic principles regulating the chartering, composition and decision-making of such GAs. If these are successful in Europe, their norms may diffuse to other world regions.
\end{abstract}

\title{
Design/methodology/approach
}

This is a conceptual article which presents principles for international governance for sustainability based on current experiences with governance in the European Union.

\section{Findings}

The article points out that since the publication of the Brundtland report, among the most significant political/institutional changes with regard to policies for environmental sustainability have been the expansion of the competences of the European Union (EU) and the emergence of a numerous and wide-ranging set of trans-national non-governmental organizations. The article indicate show this new institutional terrain may be used to forge new governance strategies for sustainability.

\section{Originality/value}

The originality/value of the article lies in its analysis of decision-making in the $E U$ as a point of departure for global governance for sustainability.

Keywords: Governance - European Union - sustainability

\section{INTRODUCCIÓN}

En el momento en que el Informe Brundtland sobre desarrollo sostenible fue publicado (WCED, 1987), la suposición obvia fue que ésta era la tarea de políticos actuando en nombre de Estados nacionales soberanos que entrarían en tratados intergubernamentales unos con otros. Debido a que el alcance de la mayoría de los problemas que estaban siendo considerados era global, se asumió además que estos tratados debían ser tan comprehensivos como fuera posible y esto implicaba un rol privilegiado para las Naciones Unidas y sus organismos especializados. Y, de hecho, el Informe parece haber servido como un estímulo para la convocatoria de una serie de conferencias mundiales en las cuales dichos tratados fueron redactados. (Y sigue haciéndolo, siendo la más reciente la Cop 15, la Conferencia de las Naciones Unidas sobre Cambio Climático en Copenhague en diciembre del 2009). Los signatarios de estos proyectos de convenciones se suponía que los ratificarían con prontitud y los implementarían fielmente de acuerdo con la bien establecida doctrina de pacta sunt servanta.

Los siguientes 20 años han demostrado que esta estrategia es defectuosa. Algunos países firman tratados, pero no los ratifican (incluyendo países importantes como los 
Estados Unidos). Otros sí los ratifican, pero no cumplen con sus obligaciones. Y no pocos no toman partido hasta que el resultado del tratado se ha tornado más claro. Las cumbres mundiales y las negociaciones de tratados continúan celebrándose, pero con expectativas reducidas.

Afortunadamente, la política mundial ha cambiado en forma dramática desde Brundtland. Rasgos que eran apenas discernibles a mediados de los 80 se han convertido en importantes tendencias. Un gran número de gobiernos autocráticos han colapsado y han sido reemplazados por gobiernos democráticos. Los actores no estatales han crecido en importancia y ahora habitualmente trascienden las fronteras nacionales; los públicos domésticos se han dado cuenta y se están movilizando en torno a problemas externos como nunca antes (Ruggie, 2004); las corporaciones globalizadas han adquirido recursos superiores a todos a excepción de unos pocos Estados soberanos y están operando de manera simultánea y en forma relativamente autónoma en muchos de ellos; comunidades científicas transnacionales se han formado alrededor de una multitud de temas especializados y están ejerciendo una mayor influencia sobre la elaboración de políticas en todos los niveles del territorio; el costo de la comunicación internacional y del transporte ha caído precipitadamente; los flujos masivos de personas a través de las fronteras nacionales y continentales han revelado lo difícil que ha llegado a ser para los gobiernos controlar incluso un elemento central de su soberanía; una ideología permisiva del laissez-faire y el laissez-passer en bienes y servicios (pero no en personas) ha proliferado más allá de su anterior centro liberal angloamericano. Finalmente, los asuntos múltiples y complejos que involucran el desarrollo sostenible se han vuelto más salientes para los públicos masivos y mejor apoyados dentro y a través de las comunidades científicas. En otras palabras, el argumento genérico en Brundtland de que la sostenibilidad debería ser considerada como una preocupación urgente que trasciende las fronteras nacionales existentes y sobrepasa las capacidades estatales actuales ha ganado. Lo que se necesita es una estrategia común que tome en cuenta la complejidad del problema y los cambios políticos trascendentales de los pasados 20 años.

La Unión Europea (UE) ofrece una interesante arena para la gobernabilidad emprendedora orientada a la sostenibilidad. La UE no puede gobernar en el sentido tradicional de la aplicación jerárquica de la autoridad y el castigo a los transgresores, como un Estadonación consolidado. Para superar este déficit ha desarrollado una práctica elaborada y una justificación normativa para sí misma en tanto "sistema de gobernabilidad de múltiples niveles y policéntrico" que puede contener interesantes lecciones para la gobernabilidad internacional para la sostenibilidad en el futuro. Ninguna de las otras instituciones regionales del mundo -MERCOSUR, ASEAN, CIS, OAS, AU y más de cien otros acrónimos- merece este rótulo. A lo más ellos son instrumentos para la cooperación regional, no para la integración.

El sistema de gobernabilidad de múltiples niveles y policéntrico de la UE tiene varias características: es gobernabilidad más que gobierno; es parcial más que comprehensivo, y es regional más que global. 


\section{GOBERNABILIDAD MÁS QUE GOBIERNO}

La gobernabilidad no opera a través de instituciones monopólicas formales que ejercen la máxima autoridad sobre un territorio específico, esto es, Estados, pero sí a través de acuerdos informales de actores que ejercen algún grado de control sobre diversas funciones, es decir, redes. Tales acuerdos están abiertos a la participación de diferentes tipos de actores -públicos y privados, con y sin fines de lucro, nacionales y transnacionales, expertos y aficionados, productores y consumidores, grandes y pequeños-, quienes han sido identificados y se han aceptado los unos a los otros en tanto "partes interesadas" (stakeholders). Lo que estos actores tienen en común es una mayor conciencia de la interdependencia. Tienen objetivos contrapuestos, pero dependen lo suficiente los unos de los otros de modo que nadie puede simplemente imponer una solución a los demás; y todos perderían si no se encontrara una solución. Ellos saben que sus respectivas contribuciones son necesarias en diversos grados si algún problema ha de resolverse o si algún bien público ha de ser producido. Más aún, también saben que la solución no puede simplemente ser comprada en el mercado o comandada por el gobierno. Estos otros dos mecanismos de distribución normalmente serían preferidos por razones prácticas o ideológicas, pero gracias ya sea a una falla del me $<<<$ rcado o del Estado (o de ambos), las soluciones de gobernabilidad más complejas que están en segundo lugar pasan a ser las mejores.

Es precisamente su informalidad tanto en su composición como en su funcionamiento lo que hace que los acuerdos de gobernabilidad sean más apropiados como punto de partida para abordar los asuntos de sostenibilidad. Pueden ser "constituidos" en sus inicios por prácticamente cualquier nivel de gobierno o incluso por un conjunto de instituciones privadas, pero lo que es especialmente importante es que la pertenencia a ellos no depende de la igualdad formal (e ilusoria) de las organizaciones intergubernamentales y de los productos de la toma de decisiones por consenso negociado; no por unanimidad o votación. Además, los actores en dichos acuerdos pueden en forma colectiva fijar los estándares y establecer sistemas de monitoreo sin tener que pasar por procesos formales de ratificación. Idealmente, tales acuerdos deberían "hacerse cumplir a sí mismos", pero en el mundo real su papel está siempre circunscrito tanto por los mecanismos de mercado como por los estatales. Con el fin de alcanzar un consenso en primer lugar, las "partes interesadas" a menudo dependen de su mutuo temor de que, si los mercados estuvieran para distribuir bienes o los Estados para imponer soluciones, estarían todos peor. La implementación de opciones hechas por la gobernabilidad puede ser aún más problemática. Los mercados pueden ofrecer incentivos poderosos para abandonarlas y, de este modo, ganar una ventaja competitiva de corto plazo. La falta de apoyo oficial de los gobiernos puede significar que los aparatos de vigilancia sean débiles y las sanciones imposibles de aplicar.

En otras palabras, los acuerdos de gobernabilidad en Europa pueden encajar con las condiciones políticas de un mundo "Post-Brundtland" que ha perdido sus claras demarcaciones de soberanía nacional, que tiene que hacer frente a problemas que superan las especializaciones funcionales arraigadas, que ha generado una amplia variedad de actores que insisten en su derecho a participar y que aún no ha producido un sistema de gobierno 
regional. No son, sin embargo, la respuesta definitiva al desafío del desarrollo sostenible. En el futuro previsible, tales acuerdos todavía tendrán que afrontar en última instancia la prueba de la competencia del mercado y confiar en la legítima coerción de las autoridades estatales nacionales. No pueden mantenerse solos.

\section{PARCIAL MÁS QUE COMPREHENSIVO}

El desarrollo sostenible no demanda nada sino amplitud. Requiere que los dominios múltiples y relativamente autónomos del quehacer humano tengan que estar coordinados. El problema es que los acuerdos de gobernabilidad funcionan mejor cuando son parciales, esto es, cuando las partes interesadas involucradas son relativamente pocas en cantidad y altamente dependientes las unas de las otras. Estos acuerdos pueden ser útiles a la hora de superar los límites "intergubernamentales" impuestos por la soberanía nacional, pero, ¿cuán efectivos pueden ser en tender un puente entre las diferencias en conocimiento e interés incrustadas en distintos dominios funcionales?

La respuesta simple es que no lo sabemos. Sólo la innovación y la experimentación nos permitirán enterarnos. ¿Pueden las partes interesadas en un dominio -propietarios, empleados, expertos, representantes de intereses y funcionarios públicos- aprender de lo que otros han logrado? ¿Percibirán siquiera los participantes comprometidos en resolver sus problemas de sostenibilidad los efectos positivos y negativos que sus esfuerzos tienen sobre otros? ¿Emergerán especialidades científicas enteramente nuevas para lidiar con los intersticios entre diferentes acuerdos de gobernabilidad? ¿Puede uno siquiera imaginar algo así como "fusiones y adquisiciones" en el futuro en las que acuerdos de gobernabilidad inicialmente separados se combinen para hacer frente a asuntos más comprehensivos?

La historia a nivel nacional en las sociedades más desarrolladas sugiere que esto fue posible. Los "derrames" ocurrieron habitualmente a través de especialidades científicas y funciones administrativas. Algunas arenas de política probaron ser estratégicamente más importantes que otras e incorporaron a las menos innovadoras o a las más débiles. Lo que hizo una contribución especialmente significativa a estos procesos de difusión y expansión fue la existencia dentro de cada Estado nacional de algo llamado "sociedad civil". Los participantes en la mayoría de los acuerdos de gobernabilidad no han sido individuos sino organizaciones. Y estas sociedades científicas, asociaciones profesionales y de negocios, sindicatos, movimientos sociales, organizaciones comunitarias y otras se tienen que relacionar entre sí a través de niveles y arenas. A menudo compiten por la afiliación de miembros y por apoyo financiero, y tienen que formar alianzas para propósitos más amplios. Sin embargo, una peculiaridad especial de este proceso en el nivel nacional está prácticamente ausente en el nivel transnacional, a saber, la existencia de un sistema de partidos políticos competitivo. Con sus apelaciones ideológicas, campañas electorales y su rol comprehensivo en la formación de gobiernos, estos partidos -en el contexto de un Estado-nación- proveyeron un poderoso incentivo para conectar las "parcialidades" incrustadas en dominios de política separados. Como podremos ver más adelante, sólo en unas pocas regiones transnacionales algo equivalente a esto todavía existe. 
Confiar en la gobernabilidad de dominios de política funcionalmente diferenciados para lograr el desarrollo sostenible es definitivamente paradójico. Propone el uso de un instrumento parcial para alcanzar un objetivo comprehensivo. A lo más ofrece la promesa de que los esfuerzos dispersos en la resolución funcional de problemas por parte de grupos aislados de interesados no sólo no interferirán los unos con los otros, sino que eventualmente conducirán a una aproximación más abarcativa. Sin embargo, la insostenibilidad está aumentando a un ritmo rápido y algo se tiene que hacer más temprano que tarde. Por supuesto, un sistema multisectorial más comprehensivo para evaluar los riesgos y asignar las respuestas sería preferible. Por desgracia, no estará disponible en un futuro previsible.

\section{REGIONAL MÁS QUE GLOBAL}

No sólo el desarrollo sostenible debería ser comprehensivo en su sustancia funcional, sino que también debería ser global en su alcance territorial. Su principio más básico es que el mundo ha llegado a ser uno solo. Existe únicamente un ecosistema y una economía cada vez más integrada. La conciencia de esto ha aumentado, pero el mundo no está más cerca de poseer un mecanismo político a nivel global para identificar las prioridades de política, establecer estándares relevantes, recaudar los recursos necesarios, apoyar los esfuerzos colectivos y, cuando todo lo demás falla, castigar las deserciones del bien común. Los Estados nacionales todavía tratan de hacer esto y necesariamente lo hacen de una forma altamente desigual y autorreferente.

Pero ya no se encuentran solos. Uno de los cambios más significativos que ha ocurrido desde Brundtland ha sido el surgimiento de "regiones transnacionales". Entre lo global y lo local en algunas partes del mundo -y en ningún lugar más que en Europa- existen ahora complejos sistemas políticos "de múltiples niveles" en los cuales la responsabilidad por el uso de la autoridad pública es compartida a través de diferentes niveles territoriales y la implementación de políticas requiere de la cooperación constante entre ellos. Estamos convencidos de que, dado los decepcionantes resultados de tratar de alcanzar acuerdos mundiales y empoderar a las organizaciones intergubernamentales globales, este nivel "regional" intermedio nos provee de otra "segunda mejor" alternativa estratégica.

Y el hecho de que la UE -ahora expandida para incluir a 27 países- esté a la vanguardia de tales desarrollos es especialmente alentador. Esta región del mundo tiene los recursos colectivos -materiales y humanos- para hacer una contribución altamente significativa por su propia cuenta. La existencia de una organización supranacional con una capacidad probada para generar beneficios para todos sus miembros y de una población que es más consciente que casi cualquier otra de los costos involucrados en un "desarrollo no sostenible" son más que casuales. Los ciudadanos de la UE únicamente esperan que su organización regional haga algo en relación a estos riesgos (Schmitter, 1996). Otro factor significativo es que Europa -dentro y más allá de la UE- ya posee los elementos clave de una amplia sociedad civil continental que puede desempeñar un papel crucial en la identificación de las prioridades, en la difusión de mejores prácticas y en la presión para que haya "derrames" de un dominio a otro. Esta región aún no tiene un sistema de partidos supranacional distintivo, 
pero tal sistema podría emerger en un futuro cercano y los temas de sostenibilidad bien podrían determinar su configuración.

Los estudiantes de relaciones internacionales han reconocido hace largo tiempo que el cambio voluntario y pacífico en este nivel macro requiere del ejercicio del liderazgo (ellos lo Ilaman "hegemonía"). Algún actor tiene que correr los inicialmente mayores riesgos y pagar originalmente los costos más elevados. El imperio permite la imposición de tales cambios por la fuerza; los mayores vuelcos de política dentro de las políticas nacionales típicamente siguen de cambios en el partido o la alianza en el poder. Las organizaciones regionales se encuentran en una posición única para desempeñar este rol de manera constructiva, precisamente cuando no son una simple fachada cubriendo la dominación del Estado nacional más poderoso, la firma privada más grande, el partido político momentáneamente hegemónico o la organización no gubernamental mejor financiada. La UE ha demostrado en forma repetida su independencia de la hegemonía de sus Estados miembros más grandes y su capacidad para ejercer influencia fuera de su propia esfera territorial. Ninguna otra organización regional transnacional puede (aún) hacer esa reivindicación, razón por la cual en este momento la UE ofrece al mundo su mejor alternativa estratégica para avanzar en el desarrollo políticamente sostenible.

Mientras sea concebible que otras regiones del mundo puedan eventualmente imitar sus instituciones (de hecho, la UE ha dedicado recursos considerables a esfuerzos por clonarse y se reúne periódicamente con sus "contrapartes" en Asia, América Latina y África), lo mejor que se puede esperar en el futuro cercano es que los Estados nacionales individuales decidan que van a adoptar las regulaciones y estándares desarrollados por los acuerdos de gobernabilidad de la UE. Demás está decir que es más probable que esto suceda en los Estados "vecinos" al Sur y al Este donde tal "eurocompatibilidad" puede mejorar sus perspectivas para una eventual membrecía.

\section{COMBINANDO GOBERNABILIDAD Y LEGITIMIDAD EN LA UNIÓN EUROPEA}

Los acuerdos de gobernabilidad deben en última instancia combinar desempeño con legitimidad. Con su Libro Blanco sobre Gobernabilidad (2001), la UE literalmente proclamó su intención de poner en juego su futura legitimidad en la aplicación exitosa de acuerdos de gobernabilidad para resolver los conflictos de intereses entre sus Estados miembros y satisfacer las expectativas normativas a través de sus públicos nacionales. Al hacerlo, también reconoció implícitamente que no podía competir sobre el criterio estándar de legitimidad con las democracias nacionales. Cualesquiera modificaciones que pudieran ser introducidas en sus reglas y prácticas -incluyendo aquellas en el proyecto de Tratado Constitucional y su versión abreviada de Lisboa- no serían suficientes para convencer a la mayoría de sus ciudadanos de que la UE podría funcionar como un régimen democrático-constitucionalelectoral-parlamentario-representativo-liberal "realmente existente". Algo más tenía que justificar por qué las decisiones de esta política regional transnacional inevitablemente compleja y remota eran legítimas y provechosas de ser obedecidas. Y la "gobernabilidad" fue elegida para cumplir con este propósito. 
Sin embargo, mucho de lo que está sucediendo regularmente dentro de la UE es más el resultado de la conveniencia, del arreglo pragmático, de las presiones de tiempo, de la difusión de las "mejores prácticas", de soluciones ad hoc e incluso ad hominem que de principios compartidos y de un diseño explícito. Es todavía mi (no probada) presunción que si la UE elaborara y defendiera una serie de principios claros y diseñara sus acuerdos de gobernabilidad acorde con ellos, esto mejoraría la legitimidad de sus decisiones en el largo plazo y, sólo quizás, convertiría a la UE de un consumidor de legitimidad nacional a un productor de un nuevo tipo de legitimidad supranacional que podría aplicarse a las tareas de garantizar la sostenibilidad ecológica y social en el nivel regional.

Las siguientes secciones esbozan algunos principios de diseño centrales para los acuerdos de gobernabilidad, enfocándose tanto en la legitimidad como en la eficiencia. Esto incluye la constitución, composición y la toma de decisiones.

\section{PRINCIPIOS DE DISEÑO PARA LOS ACUERDOS DE GOBERNABILIDAD}

Si uno acepta la siguiente definición genérica: la gobernabilidad es un método o mecanismo para lidiar con una amplia variedad de problemas o conflictos en los cuales los actores con regularidad llegan a decisiones mutuamente satisfactorias y vinculantes mediante la negociación de los unos con los otros y la cooperación en la implementación de estas decisiones, entonces hay tres tareas que deberían cumplirse si tales acuerdos han de ser considerados como legítimos por aquellos que van a verse afectados por sus decisiones: (1) tienen que ser establecidos por alguna autoridad reconocida y preexistente y se les debe dar un mandato específico dentro del cual operar (constitución); (2) los actores que participan regularmente en ellos y que se presume representan a públicos más amplios tienen que ser elegidos (composición), y (3) estos actores tienen que ser instruidos acerca de cómo negociar, lograr acuerdos consensuados sobre políticas y cómo implementarlos (toma de decisiones). Lo que es crítico en relación a estas tres dimensiones es que las soluciones que requieren no corresponden a las normas que prevalecen en la mayoría de los gobiernos democráticos liberales "realmente existentes". Los acuerdos de gobernabilidad no son autoestablecidos "por la voluntad del pueblo"; no están abiertos a la participación de todos los ciudadanos, sino sólo a un seleccionado y privilegiado subconjunto de ellos, y no toman sus decisiones sobre la base de la votación de mayoría, o incluso de la votación en absoluto. Incluso menos convencional es el hecho de que estos acuerdos de gobernabilidad regionales puedan ser policéntricos y, por tanto, no estar sujetos al control jerárquico o soberano de una sola institución tal como el Parlamento o el Poder Ejecutivo.

\subsection{Constituyendo acuerdos de gobernabilidad}

En primer lugar, uno debería comenzar con la noción de constitución (chartering), esto es, de cómo un acuerdo de gobernabilidad (en adelante AG) se establece a nivel regional para hacer frente a una tarea particular. Esta pregunta de "¿por qué estos actores están tomando decisiones sobre este asunto?" debería resolverse a través de una delegación explícita de 
autoridad desde una institución preexistente legítima, es decir, por medio de una escritura de constitución (charter).

Esta noción de escritura de constitución descansa en la presunción de que un asunto o arena de política particular es "apropiada" para tal acuerdo, ergo, no es mejor manejada por una buena competencia de mercado o regulación gubernamental, y que (subsidiariedad obliga) debería abordarse al nivel supranacional. Lo que tiene que ser demostrado y defendido es la noción de que algún conjunto particular de actores, cada uno actuando autónomamente, es capaz de tomar decisiones que resolverán los conflictos involucrados y proporcionarán los recursos necesarios para lidiar con el asunto predesignado por su escritura de constitución. Es más, estas decisiones una vez implementadas serán aceptadas como legítimas por aquellos que no participaron y que han sufrido o disfrutado de sus consecuencias. Y, si esto no fuera suficiente, un AG exitoso también tendría que demostrar que su capacidad para resolver conflictos y proveer recursos es superior a cualquier cosa que un acuerdo nacional o subnacional pudiera haber hecho. Visto desde esta perspectiva, ipodría no haber demasiadas arenas de política que debieran adquirir "sus" respectivos AGs!

\subsection{Seis principios para constituir AGs}

1. El principio de la "autoridad mandatada": Ningún AG que no tenga un mandato claro y circunscrito que le sea delegado por una institución apropiada debería ser establecido. Cualquier institución de la UE debería tener el derecho de recomendar la formación y diseño inicial de un AG, es decir, su escritura de constitución, su composición y sus reglas, pero (siguiendo las disposiciones del Tratado de Roma) sólo aquellos aprobados por la Comisión deberían ser establecidos de manera efectiva, estén o no posteriormente dotados de personal, financiados, "emplazados" y/o supervisados por la Comisión. Los que no participan pero son afectados por un AG deberían tener la confianza de que éste ha sido establecido por una autoridad que ellos reconocen como legítima y que su mandato es públicamente proclamado y justificado.

2. El principio del "ocaso": Ningún AG debería ser constituido por un período indefinido, independiente de su desempeño. Mientras que es importante que los participantes en todos los AGs debieran esperar interactuar entre sí sobre una base regular e iterativa (y es importante que el número e identidad de los participantes permanezca lo más constante como sea posible), cada AG debería tener una fecha preestablecida en la cual expirar. Por supuesto, si la institución de la UE que delegó su existencia está de acuerdo de forma explícita, su escritura de constitución puede ser renovada y extendida, pero de nuevo sólo por un periodo definido. Nuevamente los ciudadanos que en el mejor de los casos son representados de manera indirecta y desigual deberían tener confianza en que estos acuerdos entre tomadores de decisiones privilegiados no los perpetuarán en el poder más allá del período necesario para el cumplimiento de su mandato. Además, ya que los AGs a menudo se arrogan a sí mismos un rol en el monitoreo de la implementación posterior de sus decisiones, la tentación de la autoperpetuación puede ser especialmente fuerte. 
3. El principio de la "separabilidad funcional": Ningún AG debería ser constituido para cumplir una tarea que no está suficientemente diferenciada de otras tareas que ya están siendo logradas por otros AGs y que no pueda ser factiblemente alcanzada mediante su deliberación y decisión independiente. Aquí, en Europa, la cuestión es la de la "policentralidad". La UE no tiene ninguna constitución o un mecanismo democráticamente responsable para prevenir la proliferación de sus AGs (Banchoff y Smith, 1998). De hecho, con tantos Estados miembros "soberanos" y tan poca autoridad centralizada, existe una tendencia inevitable a resolver los conflictos a través de compromisos que en consecuencia producen instituciones adicionales para ser distribuidas. Algún principio tiene que ser introducido para resistir esta tentación, aunque cómo se puede hacer cumplir y por quiénes no resulta evidente.

4. El principio de la "suplementariedad": Ningún AG debería ser constituido (o permitírsele el cambio de sus tareas) en una vía que duplique, desplace o incluso amenace las competencias de las instituciones regionales existentes. Los AGs europeos no son substitutos del gobierno europeo, sino que deberían ser diseñados para complementar y, por ende, para mejorar el desempeño de la Comisión, del Consejo y del Parlamento. De nuevo, la raíz del asunto es que la UE no es un Estado y no tiene (todavía) un régimen constitucionalizado y, por lo tanto, carece de los medios legales para sancionar la intrusión de los AGs sobre las instituciones gobernantes. Además, en una democracia nacional establecida se podría esperar de los partidos políticos rivales que plantearan tales asuntos de intrusión -en especial sobre las prerrogativas de los parlamentos-, pero la UE carece de ese sistema y el Parlamento Europeo prácticamente no tiene poderes para regular o limitar los poderes de sus AGs.

5. El principio de la "variedad necesaria": Cada AG debería ser libre-dentro de los límites impuestos por su escritura de constitución- para establecer los procedimientos internos que sus participantes estimen apropiados para cumplir la tarea que le fue asignada. Dada la diversidad inherente a estas tareas diferenciadas funcionalmente, es de esperar que los AGs adopten una amplia variedad de formatos distintivos para definir su programa de trabajo, su criterio de participación y sus reglas para la toma de decisiones -mientras (esperablemente) se conforma a principios similares de diseño general-. Uno de los argumentos más convincentes de los primeros pensadores funcionalistas como David Mitrany es el de que los representantes y expertos que son hechos responsables de resolver los problemas particulares que trascienden los Estados nacionales deberían ser autónomos en la elección de los medios y estrategias organizacionales para hacerlo, dentro del mandato dado a ellos inicialmente. Por consiguiente, se sigue de la variedad de asuntos con los que se tiene que lidiar al nivel regional que las reglas y los recursos para hacerlo deberían ser correspondientemente variados.

6. El principio del "borde alto" o del "antiderrame": Ningún AG debería ser autorizado por su institución mandante para exceder las tareas que le fueron delegadas originalmente. $\mathrm{Si}$, como sucede a menudo en el curso de las deliberaciones, un AG concluye que no puede cumplir con su mandato original sin asumir nuevas tareas, debería exigirse obtener 
un cambio específico en su mandato a fin de hacer esto. De acuerdo con la teoría neofuncionalista de la integración regional, uno tiene todos los motivos para sospechar que los funcionarios, expertos y representantes de intereses que se agrupan alrededor de un AG dado harán todos los esfuerzos por ampliar sus tareas, tanto en su alcance como, tal como hemos visto anteriormente, en tiempo. Si no lo hicieran, el proceso se estancaría en un conjunto de instituciones "autoencapsuladas" que poca o ninguna contribución hacen al proceso general de integración. Paradójicamente, en aras de la legitimidad, el diseño de los AGs debería resistirse a estos esfuerzos de "intercambio de favores" y "concesiones mutuas". Las decisiones que impliquen la negociación de tales intercambios a través de áreas temáticas circunscritas deberían ser competencia de otras instituciones regionales, esto es, la Comisión, el Consejo de Ministros, el Consejo Europeo y, esperablemente en el futuro, el Parlamento Europeo en el caso de la UE; no un AG altamente especializado.

\subsection{Componiendo acuerdos de gobernabilidad}

Una vez que el AG ha sido constituido, ahora debe ser compuesto, es decir, aquellos que tienen derecho a participar en él deben ser seleccionados (y no elegidos). Ya sean especificados ex ante en la escritura de constitución o seleccionados ex post por algún cuerpo con autoridad, estas personas (o, mejor dicho, estos representantes organizacionales) deberían tener alguna razón justificada para ser incluidos en las negociaciones y deliberaciones y para formar parte del (anticipado) consenso. La palabra clave en el actual debate en torno al concepto de gobernabilidad es la de partes interesadas. A diferencia de un gobierno democrático donde se presume que todos los ciudadanos tienen el mismo derecho a participar, en los AGs sólo un subconjunto de estos ciudadanos, esto es, aquellos que han expresado una mayor preocupación o que se considera que es más probable que sean afectados, pueden participar. El cálculo parece ser que si las partes interesadas pueden alcanzar un consenso respecto de lo que tiene que hacerse y, aún más, si continúan estando de acuerdo en cómo implementar lo que ha sido elegido, sus conciudadanos se someterán como si ellos mismos hubieran tenido la oportunidad de participar.

\subsection{Cuatro principios para componer AGs}

1. El principio del "umbral mínimo": Ningún AG debería tener más participantes activos que los que son necesarios para el propósito de cumplir con su tarea encomendada. Tiene el derecho autónomo de buscar información y pedir consultoría de cualquier fuente que elija; sin embargo, para el proceso actual de redacción de probables políticas y de decisión acerca de ellas, sólo aquellas personas u organizaciones consideradas capaces de contribuir a la gobernabilidad de la tarea designada deberían participar. La decisión por consenso requiere, como mínimo, de tres cosas: (1) un número restringido de participantes debido a que el acuerdo se torna exponencialmente más dificultoso de alcanzar a medida que crecen en número; (2) aquellos que participan deberían poseer algún tipo o grado de "especificidad de activos" que significa recursos materiales, intelectuales o políticos 
que son apropiados para las tareas a cumplir, y (3) como personas u organizaciones, los participantes deberían tener la capacidad no sólo de representar categorías relevantes sino también de entregar su conformidad a cualesquiera decisiones que sean tomadas. Restringir su número es un prerrequisito para las otras dos.

2. El principio del "interés": Ningún AG debería tener, como participantes activos, a personas u organizaciones que no tengan un interés significativo en los asuntos que rodean a la tarea que le fue asignada. Debería considerarse que los conocedores (expertos) que se especializan en tratar con la tarea tengan un interés, incluso si profesan no representar los intereses de ninguna parte interesada en particular. Esto sigue de la discusión anterior acerca de la "especificidad de activos". Huelga decir que el definir qué grado o tipo de activo constituye "un interés" y quién controla ese interés está destinado a ser políticamente controvertido, ya que -gracias a la creciente interdependencia de los dominios de política- el número de representantes y expertos que pueden hacer tal reclamación es potencialmente ilimitado. Como una línea directiva aproximada, una parte interesada relevante podría ser definida como una persona u organización cuya participación es necesaria para la toma de una decisión por consenso (potencialmente) vinculante y cuya colaboración es necesaria para la implementación exitosa de dicha decisión. En la práctica, es probable que esto esté determinado sólo por un proceso iterativo en el cual aquellos inicialmente excluidos del AG hacen suficientemente conocidas sus demandas a las partes interesadas y a los poseedores de conocimiento, de forma que son posteriormente incluidas.

3. El principio del "Privilegio Europeo": En iguales condiciones, los participantes en un AG ligado a la UE deberían representar circunscripciones a lo largo de toda Europa. Concedido que, en la práctica, estos representantes pueden tener que confiar fuertemente en el personal y financiamiento nacional e incluso subnacional y pueden ser dominados por cálculos de interés nacionales y subnacionales, y concedido que mientras más grande sea la circunscripción en números, escala territorial y diversidad cultural más difícil puede ser adquirir la especificidad de activos que provee la base para la retención de intereses, sin embargo, la característica distintiva de un AG europeo depende del privilegio de este nivel de agregación en la selección de participantes. Esto se sigue del argumento inicial de que sólo los AGs regionales más que los globales son viables en este punto del tiempo. Dada la naturaleza "inconclusa" de la integración europea, pueden haber partes interesadas y poseedores de conocimiento significativos en potenciales Estados miembros e incluso en aquellos que han elegido de manera explícita no unirse a la UE pero que se ven fuertemente afectados por sus políticas.

4. El principio del "adversario": Los participantes en un AG deberían ser seleccionados para representar circunscripciones que se sabe que tienen intereses diversos y, especialmente, opuestos. Ningún AG debería ser compuesto de una preponderancia de representantes que son conocidos por tener una posición similar o que ya han formado una alianza para un propósito en común. En el caso de los "conocedores" de los que se presume que no tienen circunscripciones sino ideas, ellos deberían ser elegidos para representar 
cualesquiera diferentes teorías o paradigmas que puedan existir con respecto a una tarea particular. Para respetar este principio, puede ser necesario para los diseñadores de tales acuerdos el jugar un rol proactivo en ayudar a los intereses peor dotados o más dispersos de tal modo de que se organicen y se motiven lo suficiente para participar contra sus adversarios "privilegiados". Este elemento de "patrocinio", si bien tiene la intención de fomentar un mayor equilibrio en las relaciones antagónicas, puede entrar en conflicto con el principio subsecuente de la igualdad "putativa" de trato y estatus. También puede generar serios cuestionamientos en lo concerniente a la autonomía de tales organizaciones "patrocinadas". Lo que es crucial sin embargo acerca de este aspecto de la composición de los AG es que los no-participantes tengan confianza de que sus deliberaciones y negociaciones incluyen un rango suficientemente amplio de participantes y opiniones actuales. Sólo entonces estos outsiders creerán que han sido representados indirectamente en sus actividades y acordarán legitimidad a sus decisiones.

\subsection{Toma de decisiones en los acuerdos de gobernabilidad}

Ahora que el AG ha sido constituido y compuesto, debe tomar e implementar decisiones. Como hemos visto arriba, las reglas habituales que dominan las organizaciones intergubernamentales (unanimidad) o las federaciones democráticas (mayoría simple o calificada) no son apropiadas. Más bien, una "metanorma" deliberadamente vaga debería aplicar, a saber, el consenso. Pero ¿cuáles son los principios operativos que enmarcan este proceso de formación de consenso?

\subsection{Siete principios para la toma de decisiones en los AGs}

1. El principio de la igualdad "putativa": Todos los participantes en un AG deberían ser considerados y tratados como iguales, incluso cuando representen circunscripciones muy diferentes en tamaño, recursos, estatus público o privado, e "influencia política" en el nivel nacional. Ningún AG debería tener participantes de segunda y tercera clase, aunque sea necesario distinguir sin ambigüedades entre quienes pueden participar y quienes sólo son consultados. Los AGs no son "democráticos", por lo menos, no como se define convencionalmente. No tienen sistemas de partidos competitivos, elecciones periódicas o mecanismos de votación, pero existe una forma en la que pueden adquirir algo que se aproxima a la legitimidad democrática. Si sus participantes se tratan los unos a los otros como iguales -como cuasiciudadanos- esto debería mejorar la calidad de sus deliberaciones por medio del estímulo de una expresión más honesta de preferencias y del desarrollo de relaciones de mutua confianza entre ellos. Siempre y cuando un consenso eventual sea alcanzado, será mucho más probable que sea respetado voluntariamente tanto por los miembros como por los no miembros.

2. El principio de interacción horizontal: Debido a la presunción de igualdad entre los participantes, los procesos de deliberación y de toma de decisiones internos de un AG deberían evitar tanto como sea posible tales dispositivos jerárquicos internos 
como la delegación estable de tareas, distinciones entre expertos "neutrales" y representantes "comprometidos", estructuras de liderazgo formalizadas, acuerdos de deferencia, etc., y deberían estimular la flexibilidad en el cumplimiento de las tareas colectivas, acuerdos rotatorios para el liderazgo y relatoría, deliberación verbal extensiva, junto con una atmósfera general de informalidad y respeto mutuo. Aquí, la lógica es similar a aquella de la igualdad presunta. Mientras más débil sea una parte interesada dada en la sociedad o en la política, más fuerte debería ser su rol dentro del AG, al menos relativamente hablando. En la UE, esto ha sido resuelto a través de la sobrerrepresentación sistemática de los Estados miembros pequeños en tales acuerdos y de la voluntad demostrada de los más grandes de no hacer valer su hegemonía.

3. El principio del consenso: Las decisiones en un AG deberían ser tomadas por consenso más bien que por votación o por imposición. Esto implica que ninguna decisión puede ser tomada contra la oposición expresada de cualquier participante, aunque los mecanismos informales usualmente permiten que los actores se abstengan en un determinado tema o expresen públicamente opiniones disidentes sin el ejercicio del veto. En otras palabras, el consenso no necesariamente implica unanimidad. Ni qué decir que los dispositivos primarios para llegar a un consenso son la deliberación (es decir, tratar de convencer a los adversarios de lo bien-fundada de la propia posición), el compromiso (es decir, mediante la aceptación de una solución en algún lugar entre las preferencias expresadas por los actores) y el ajuste (esto es, a través del peso de la intensidad de las preferencias de los actores). La interacción regular e iterativa entre un conjunto estable de representantes también es importante, ya que esto permitiría el establecimiento de "intercambios" a través de una sucesión de asuntos controversiales. Quizás, el aspecto más importante para la formación de consensos es la prevención de la dominación acumulativa que podría emerger dentro de un AG por la vía de los votos reiterados en los cuales los mismos participantes están regularmente en el lado ganador. Además, la toma de decisiones por consenso es un incentivo poderoso para que los actores "permanezcan en el juego" en lugar de desertar después de perder.

4. El principio de la "puerta abierta": Cualquier participante debería ser capaz de salir de un AG a un costo relativamente modesto y sin sufrir represalias en otros ámbitos, ya sea por otros participantes o por autoridades nacionales o regionales. Además, un ex participante tiene el derecho de anunciar esta salida ante un público más amplio (y la amenaza de hacerlo debería considerarse un aspecto normal de procedimiento), pero no la garantía de que, por salirse, puede interrumpir unilateralmente el proceso de gobernabilidad. Esto provee el complemento para el incentivo notado arriba de permanecer en el juego. Si una sola parte interesada o una minoría de partes interesadas disienten en forma persistente de las decisiones que han surgido consensualmente entre los otros, la salida de bajo costo puede ser una alternativa importante a lo que Albert Hirschmann (1970) ha llamado "voz" o "lealtad". El prospecto de ejercer esta opción debería mantener al grupo dominante en línea; sin embargo, con impedir completamente su capacidad para avanzar hacia adelante en un asunto determinado. 
5. El principio de la proporcionalidad: Aunque sería contraproducente para las influencias ser formalmente pesadas o contadas de manera equitativa, es deseable que a través del rango de decisiones adoptadas por un AG dado haya un sentido informal de que los resultados alcanzados son aproximadamente proporcionales a los activos específicos con que cada participante contribuye (de manera diferencial) al proceso de solución de las inevitables disputas y de cumplimiento de las tareas delegadas. Una forma más ortodoxa de captar este principio sería refiriéndose a la "reciprocidad", aunque esto parece transmitir el significado de participaciones o beneficios iguales a través de algún conjunto de iteraciones. La "proporcionalidad" es similar, pero permite la probabilidad de que las inequidades estables en beneficios emerjan y sean aceptadas como legítimas sobre la base de activos y contribuciones iniciales diferenciales. En el contexto de la UE, esta noción es complementada por la de "convergencia", a saber, que con el tiempo y a través de un rango de temas, el desempeño de los participantes debería tornarse cada vez más similar al de aquel con los activos de mejor rendimiento.

6. El principio de las "alianzas cambiantes": Con el tiempo, dentro de un AG dado debería esperarse que el proceso de formación de consenso sea conducido por diferentes conjuntos de participantes y que ningún participante individual o minoría de participantes sea persistentemente requerida para hacer los mayores sacrificios a fin de alcanzar ese consenso. Otra forma de describir esta condición es por medio del "pluralismo". Siempre que su composición sea propiamente "adversarial", incluso el AG más especializado debería tener intereses múltiples y rivales dentro de él. Idealmente, estas líneas de clivajes deberían atravesar aquellas de los intereses nacionales, abriendo las posibilidades para la formación de coaliciones transnacionales. Pero incluso si ese no es el caso y los representantes de los mismos países "monolíticos" se enfrentan entre ellos una y otra vez, es de esperar que los mismos siempre ganen o pierdan. Y, si éste llega a ser el caso, todavía está disponible la opción relativamente poco costosa de la "puerta abierta" para prevenir la aparición de dominación o hegemonía de parte de los participantes mejor dotados.

7. El principio de los "pesos y contrapesos": Ningún AG debería tomar una decisión vinculante sobre personas u organizaciones que no son parte de sus deliberaciones a menos que esa decisión no pueda ser explícitamente desaprobada por otra institución que esté basada en diferentes prácticas de representación y/o de circunscripción. Esta es una de las principales razones por las que los AGs tienen tan pocas probabilidades de éxito en otras regiones o a nivel global donde no existen tales instituciones de orden superior con autoridad. En Europa, la UE desempeña esta función y normalmente y en primera instancia será esta institución que en forma inicial "constituyó" el AG la que servirá de chequeo externo de sus decisiones. Eventualmente, uno puede incluso imaginar que el Parlamento Europeo a través de la estructura de su comité interno podría acordar aumentar su rol como coaprobador de las decisiones del AG. Los pesos y contrapesos son una característica legitimadora muy importante de las unidades territoriales en todas las políticas federalistas y descentralizadas y sus actividades son normalmente supervisadas por aún otra capa de toma de decisiones, a saber, una Corte Suprema o Constitucional independiente tal como la Corte Europea de Justicia. 


\section{CONCLUYENDO CON ALGUNAS DUDAS}

Establecer un conjunto de AGs para tratar los asuntos funcionalmente diferenciados relacionados con la sostenibilidad ecológica y social al nivel de Europa no es la panacea. No funcionará para resolver todos los asuntos de política que rodean la sostenibilidad y no funcionará a menos que esté basado firmemente en principios de diseño políticos así como también administrativos. Y eso significa que las decisiones difíciles que involucran su escritura de constitución, composición y reglas de decisión no pueden ser eludidas en forma indefinida. A menos que los AGs dentro de la UE sean "apropiadamente" diseñados, no hay ninguna razón para confiar en que sus decisiones serán más sostenibles o más innovadoras que aquellas tomadas por los gobiernos nacionales o integradas en los tratados internacionales. Y, como se enfatizó con anterioridad, los AGs nunca funcionan solos sino únicamente en conjunción con las normas de la comunidad, la autoridad estatal y la competencia de mercado.

Dos dilemas clave deben abordarse, incluso si el progreso está hecho sobre las difíciles decisiones involucradas en el diseño de los AGs:

1. La proliferación de AGs regionales tiende a ocurrir dentro de arenas de política compartimentalizadas (y aún más en la UE que en sus Estados miembros) y deja sin resolver el gran problema de cómo conflictos eventuales entre sus decisiones van a ser resueltos. Los múltiples "acuerdos de gobernabilidad" en los niveles micro o medio, sin importar cuán participativos, innovadores y sostenibles sean en sí mismos, ipueden terminar generando macrorresultados que nadie esperaba y que nadie quiere!

2. Los criterios para la inclusión de participantes y la toma de decisiones en los AGs regionales no son generalmente compatibles con los estándares de legitimación democrática utilizados dentro de las políticas nacionales y subnacionales, aunque la experimentación con los AGs está ocurriendo en todos los niveles de agregación. Antes de que los AGs puedan ser confiablemente desplegados y generar un sentido de obligación entre públicos más amplios, puede ser necesario gastar un buen esfuerzo en cambiar las nociones de la gente respecto de lo que la democracia es y en lo que se está convirtiendo.

En el momento presente sólo la UE ofrece una alternativa políticamente sostenible para las políticas ambientales de los Estados nacionales y para los tratados entre ellos. Los 20 años o algo que han pasado desde el Informe Brundtland han revelado los límites de la confianza en la política nacional e internacional, al mismo tiempo que la UE ha emitido un número impresionante de directivas en estos temas. Ha alcanzado estas decisiones a través de procesos de "gobernabilidad" muy complicados, poco comprendidos y a menudo improvisados. A pesar de una buena cantidad de quejas acerca de la legitimidad de la UE misma, estas decisiones han sido aceptadas por la mayor parte de los ciudadanos europeos e implementadas por los gobiernos europeos; y hay más a la vista.

No hay una organización regional transnacional equivalente en el resto del mundo. Si mis especulaciones sobre los principios de diseño son correctas, es aún menos probable que algún acuerdo para la "gobernabilidad global" produzca legitimidad y, por ende, decisiones 
políticamente sostenibles. Lo mejor que se podría esperar sería la adopción de las directivas de la UE por parte de los gobiernos nacionales y, eventualmente, su implementación en otras regiones del mundo, pero sólo una vez que estas regiones hayan construido una capacidad institucional para lidiar con otros problemas económicos y sociales comunes. Uno no puede descartar completamente la posibilidad de que los gobiernos nacionales sean conducidos por la extrema necesidad o por impedir una catástrofe a dotar a algunos "instrumentos de gobernabilidad global" con los poderes para formular e implementar resoluciones vinculantes, pero en el momento actual y para el futuro previsible las soluciones para nuestra "insostenibilidad" global están en gran parte en las manos de los productores privados que compiten entre sí, distribuidos desigualmente a través de las políticas nacionales, variables según la industria y el sector, y todavía en disputa entre diferentes tecnologías. La única estrategia política viable que puedo prever es proceder en forma incremental (y de manera insuficiente) en los niveles parciales y regionales de acuerdo con los relativamente simples principios de la constitución, composición y decisión dentro de los AGs.

\section{BIBLIOGRAFÍA}

Banchoff, Thomas y Mitchell P. Smith (1998): Legitimacy and the European Union, Longman, London.

Comunicación de la Comisión del 25 de julio de 2001: European governance - $A$ white paper [COM (2001) 428 final - Official Journal C 287 of 12.10.2001].

Eurobarometer (2008): encuesta publicada en marzo de 2008, dos tercios de los respondientes expresaron su preferencia porque las decisiones referidas a políticas acerca del medio ambiente fueran tomadas al nivel europeo, en http://env-health.org/a/2861/ (consultado el 22 de julio de 2009).

Hirschman, Albert O. (1970): Exit, voice, and loyalty: responses to decline in firms, organizations, and states, Harvard University Press, Cambridge, Mass.

Keohane, Robert (1984): After hegemony: Cooperation and Discord in the World Political Economy, Princeton University Press, Princeton.

Schmitter (1996): "Examining the Present Euro-Polity with the Help of Past Theories", en Gary Marks, Fritz Scharpf, Philippe C. Schmitter y Wolfgang Streeck: Governance in the European Union, Sage Publications, London, pp. 1-14.

Ruggie, J. (2004): "Reconstituting the Global Public Domain-Issues, Actors, and Practices", European Journal of International Relations, Vol. 10, № 4, pp. 499-531.

World Commission on Environment and Development (WCED) (1987): Our Common Future, Oxford University Press, Oxford. 
